\title{
How Might Social Distancing Impact Gay, Bisexual, Queer, Trans and Two-Spirit Men in Canada?
}

\author{
David J. Brennan ${ }^{1} \cdot$ Kiffer G. Card ${ }^{2,3} \cdot$ David Collict ${ }^{1,4} \cdot$ Jody Jollimore ${ }^{3} \cdot$ Nathan J. Lachowsky $^{2,3}$
}

Published online: 30 April 2020

(c) Springer Science+Business Media, LLC, part of Springer Nature 2020

\section{Background}

In response to the global COVID-19 pandemic, Canadians are being asked to engage in social distancing [1]. However, potential unintended consequences of self-imposed or legally-enforced social distancing in response to COVID19 are the potential to negative impacts on mental, social, sexual, and physical health [2]. This is especially true for marginalized populations, such as gay, bisexual, queer, trans, Two-Spirit, and other men who have sex with men (GBQT2+), who already have elevated rates of mental health challenges, such as anxiety, depression, and loneliness [3-5].

Given that social support from friends, family, and partners is a known protective factor against negative mental health outcomes and sexual risk behavior among GBQT2+ [4], social distancing may exacerbate negative mental health consequences. Questions are also being raised about how social distancing will impact the sexual behavior of GBQT2+. Social and sexual networking apps have long been used by GBQT2+ to seek social connections, either virtually or in-person. Data from these applications suggest that individuals are seeking connection during the pandemic $[6,7]$. For example, compared with the previous month, Tinder has seen an increase of up to $15 \%$ on their apps in the

David J. Brennan

david.brennan@utoronto.ca

David Collict

david.collict@mail.utoronto.ca

1 Factor-Inwentash Faculty of Social Work, University of Toronto, Toronto, Canada

2 School of Public Health and Social Policy, University of Victoria, Victoria, Canada

3 Community Based Research Centre, Vancouver, Canada

4 Ontario Studies in Education, University of Toronto, Toronto, Canada
United States and up to $25 \%$ in Italy and Spain. Chat conversations are also up to $30 \%$ longer [8].

Previous research on app use among GBQT2+ indicates that dating applications provide a means of engaging in romantic, sexual and interpersonal relationships, including casual sexual encounters [9-13]. As well, these apps are tools that help address issues of mental health, internalized homophobia and low self-esteem [14]. Research is needed to understand the implications of social distancing on individuals and their sex-seeking behaviors, how individuals may be coping through dating application use, and whether individuals are continuing to engage in in-person sexual connections despite social distancing protocols.

Furthermore, it is unknown to what extent GBQT2+ may experience difficulty accessing health-related services and supplies (i.e., counselling, antiretroviral therapy,) during the COVID-19 pandemic. Indeed, services considered "non-essential" are closed or are being drastically restricted. For instance, the Hassle Free Clinic, a Toronto STI and HIV testing clinic provides services to GBQT2+ clients, has significantly reduced hours, removed clinic drop-in services, and stopped providing routine STI/HIV testing amid the pandemic [15]. Additional community-based resources providing mental and sexual health-related services to GBQT2+ have significantly limited available resources or suspended programming, including STI/HIV testing, low-cost counselling services, mental health support groups, and walk-in health services [16-18]. Across British Columbia, as a part of cancelling all non-emergency surgeries, gender affirming surgeries have largely been cancelled or postponed indefinitely [19]. These reductions in the provision of services for GBQT2+ have the potential to cause significant harm among GBQT2+ as a group that is already marginalized.

Given that social distancing measures may yet last for several months [20], and potentially even longer for older and immune-compromised GBQT2+ [21], it is important to take this opportunity to study the impact of social distancing on marginalized populations-particularly if COVID-19 
becomes a seasonal endemic disease requiring some level of social distancing among elderly and other susceptible GBQT2+ over the long term. Here we discuss data from the Community-Based Research Centre's (CBRC) Sex Now 2019-2020 Survey, a serial cross-sectional study of Canadian GBQT2 + men, to illustrate potential impacts of social distancing on these communities. Sex Now 2019 was conducted online between November 1, 2019 and February 17, 2020 and focused on the mental, social, and sexual health of GBQT2+ and their utilization of relevant services.

We recruited participants using promotional materials in French and English shared by various partner organizations across the country through newsletters, listservs, and social media platforms (e.g., Facebook, Instagram, Twitter). This included paid advertising and boosted posts on Facebook and Instagram for CBRC and partners who have a large social media presence. Additionally, several community champions and local celebrity drag queens promoted the survey on their social media profiles and were provided with a small honorarium as compensation for their time. Finally, CBRC purchased recruitment ads on popular sex seeking apps and websites (Grindr, Squirt, Scruff, Jack'd), porn sites (PornHub), and GBTQ2-oriented media sites (Xtra, Fugues). The eligibility criteria restricted participation to individuals who identified as men or another gender other than women (e.g., non-binary, genderqueer, agender; inclusive of trans men and Two-Spirit people); identified as gay, bisexual, queer, asexual, or other non-heterosexual identity (inclusive of Two-Spirit) and/or have reported having had sex with another man in the last 5 years; were at least 15 years of age; lived in Canada; provided informed consent; completed a questionnaire in either French or English; and did not already participate in this study cycle. Participants were eligible to win a $\$ 500$ gift card via random draw.

\section{Mental Health and Vulnerability}

Among the many ways that social distancing can impact the health and vulnerability of GBQT2+, we are particularly concerned of how their social and mental health might be impacted. In fact, among 6198 GBQT2+ who participated in the 2019 Sex Now Survey, over one-in-five GBQT2+ (21\%) had Patient Health Questionnaire-2 item [22; PHQ-2] depression scores greater than clinical screening thresholds and $29 \%$ self-reported having "Fair" or "Poor" mental health overall. Further, a staggering 57\% of respondents wanted help with a mental health problem they were facing. Notably, of those who wanted help with a mental health problem, 19\% wanted help dealing with suicidal thoughts. These statistics are alarming considering that we also see a dose-response relationship between the number of people GBQT2+ can turn to for support and the proportion who have depression symptoms and the proportion who want help dealing with suicidal thoughts. If social distancing prevents people from accessing these supports, it may contribute to worse mental health outcomes, potentially even suicide. In addition to the effects on social distancing, economic impacts may also harm GBQT2+ and their mental health. In Sex Now 2019, only one third (33\%) of GBQT2+ reported having "extra" money and $10 \%$ reported not being able to "make ends meet." Recent national research in Canada highlighted that half of LGBTQ2+ households (52\%, compared with 39\% of households overall) have experienced lay-offs or reduced employment as a result of the COVID-19 pandemic [23].

\section{Loss of Social Outlets}

The closures of bathhouses, gay bars, queer pop-up events, and LGBTQ Pride festival cancellations as a result of COVID-19 control measures leaves connecting online as one of the few remaining options. As a baseline, $51 \%$ of Sex Now 2019 respondents reported meeting new sexual partners in the past three months, with two-thirds of these individuals having had sex with a new partner in the past 4 weeks (and $15 \%$ of all respondents reporting sex with a new partner in the week they completed the survey). While some people, including GBQT2+, will undoubtedly continue to connect in-person with sexual partners during the COVID-19 pandemic, we know that the apps and websites are also used for online sexual acts. With $32 \%$ of GBQT2+ reporting camming or sexting online-it would suggest that at least some GBQT2+ may be able to find safe ways to fulfill their sexual needs while social distancing. It remains unclear whether these online encounters will fulfill the intimacy and connection needs of GBQT2+ men.

\section{HIV Treatment and Sexual Health}

Social distancing is likely to have additional consequences with respect to antiretroviral medication adherence and implications as a result of changes in sexual health care. Beyond issues of delayed or missed diagnoses of STIs, especially asymptomatic ones, a lack of STI screening also means that clinical eligibility for PrEP [24] will be limited if rectal STIs or syphilis are not diagnosed. Regarding PrEP, $86 \%$ of HIV-negative GBQT2 + on PrEP reported daily use. Fortunately, if access to PrEP is made difficult by increased burden on the healthcare system or if individuals are worried about accessing health services to get their prescription fulfilled these individuals might be able to switch to on demand regimens (only $11 \%$ were currently taking it on demand), but this may not provide adequate HIV prevention during sex if sexual frequency is very low. Issues with antiretroviral adherence for people living with HIV may also be impacted by a lack of physical access to healthcare providers and peer 
navigators [25]. In Sex Now, there were already indications of sub-optimal adherence among people living with HIV: $33 \%$ reported having missed a dose in the past 4 weeks alone. Disruptions to regular habits and schedules as well as potential challenges to medication access could compound these problems.

\section{Conclusion}

Control measures in response to the COVID-19 pandemic has serious potential to impact the health and wellbeing of GBQT2+. Studies conducted during this time should pay attention to impacts of social distancing on the mental, social, and sexual health of GBQT2+, as well as other marginalized populations. Online surveys with comparable methodology to that of Sex Now might be particularly beneficial in order to allow comparisons to baseline indicators. Supports to GBQT2+ organizations should be provided in order to help them adapt to the online provision of healthcare resources.

\section{References}

1. Government of Canada. Community-based measures to mitigate the spread of coronavirus disease (COVID-19) in Canada. 2020. https://www.canada.ca/en/publichealth/services/diseases/2019novel-coronavirus-infection/health-professionals/publichealthmeasures-mitigate-covid-19.html.

2. Centre for Addiction and Mental Health. Mental health and the COVID-19 pandemic. 2020. https://www.camh.ca/en/health-info/ mental-health-and-covid-19.

3. Brennan DJ, Ross LE, Dobinson C, Veldhuizen S, Steele LS. Men's sexual orientation and health in Canada. Can J Public Health. 2013;101(3):255-8.

4. Hart TA, Noor SW, Adam BD, Vernon JRG, Brennan DJ, Gardner $S$, et al. Number of psychosocial strengths predicts reduced HIV sexual risk behaviors above and beyond syndemic problems among gay and bisexual men. AIDS Behav. 2017;21(10):3035-46.

5. Herek GM, Garnets LD. Sexual orientation and mental health. Annu Rev Clin Psychol. 2007;3:353-75. https://doi.org/10.1016/j. addbeh.2016.07.011.

6. Carville O, Lanxon N. How to date online in the age of COVID19. Bloomberg. 2020. https://www.bloomberg.com/news/artic les/2020-03-20/online-dating-in-apandemic-coronavirus-keeps -singles-apart. Accessed 20 Mar 2020

7. Chau E. Dating app usage up thanks to COVID-19, study suggests. Toronto Sun. 2020. https://torontosun.com/life/relationships/datin g-app-usage-up-thanks-to-covid-19study-suggests. Accessed 14 Mar 2020.

8. Sullivan A. Love in the time of coronavirus: COVID-19 changes the game for online dating. Deutsche Welle. 2020. https://www. dw.com/en/love-in-the-time-of-coronaviruscovid-19-changes-thegame-for-online-dating/a-52933001. Accessed 27 Mar 2020.

9. Choi EPH, Wong JYH, Fong DYT. The use of social networking applications of smartphone and associated sexual risks in lesbian, gay, bisexual, and transgender populations: a systematic review. AIDS Care. 2017;29(2):145-55

10. Goedel WC, Duncan DT. Geosocial-networking app usage patterns of gay, bisexual, and other men who have sex with men: survey among users of Grindr, a mobile dating app. JMIR Public Health Surveill. 2015;1(1):E4.

11. Paz-Bailey G, Hoots BE, Finlayson T, Prejean J, Purcell DW. Trends in internet use among men who have sex with men in the United States. J Acquir Immune Defic Syndr. 2017;75(3):288-95.

12. Macapagal K, Coventry R, Puckett JA, Phillips G, Mustanski B. Geosocial networking app use among men who have sex with men in serious romantic relationships. Arch Sex Behav. 2016;45(6):1513-24.

13. Van De Wiele C, Tong ST. Breaking boundaries: the uses and gratifications of Grindr. In: Paper presented at ACM international joint conference on pervasive and ubiquitous computing. New York. 2014.

14. Grosskopf NA, LeVasseur MT, Glaser DB. Use of the Internet and mobile-based "apps" for sex-seeking among men who have sex with men in New York City. Am J Mens Health. 2014;8(6):510-20.

15. Hassle Free Clinic. Hassle free clinic will be open by appointment only. 2020. https://hasslefreeclinic.org/2020/03/17/hassl e-free-clinic-is-closed-tuesday-march-17/. Accessed 28 Mar 2020.

16. AIDS Committee of Toronto. ACT closure. 2020. https://www. actoronto.org/. Accessed 17 Mar 2020.

17. The 519. COVID-19: The 519 information and updates. 2020. https://www.the519.org/news/covid19-the519-info-updates. Accessed 17 Mar 2020.

18. Qmunity. COVID-19, important updates, March 18. 2020. https ://qmunity.ca/news/covid-19-important-updates-march-18/. Accessed 18 Mar 2020.

19. Provincial Health Services Authority (PHSA). COVID-19 update: gender affirming surgeries. 2020. https://www.phsa.ca/transcareb c/about/news-stories/2020/covid-19update-gender-affirming-surge ries. Accessed 17 Mar 2020.

20. Ferguson NM, Laydon D, Nedjati-Gilani G, Imai N, Ainsli K, Baguelin $\mathrm{M}$, et al. Impact of non-pharmaceutical interventions (NPIs) to reduce COVID-19 mortality and healthcare demand. 2020. https://www.imperial.ac.uk/media/imperialcollege/medic ine/sph/ide/gida-fellowships/Imperial-College-COVID19-NPImodelling16-03-2020.pdf.

21. CATIE. CATIE update on COVID-19. 2020. https://www.catie .ca/en/news/catie-updatecovid-19?utm_source $=$ tw\&utm_mediu $\mathrm{m}=$ socmed\&utm_campaign $=031620 \& u t m \_c o n t e n t=e n$. Accessed 17 Mar 2020.

22. Kroenke K, Spitzer RL, Williams JB. The patient health questionnaire-2: validity of a two-item depression screener. Med Care. 2003;41:1284-92.

23. Egale Canada. Impact of COVID-19: Canada's LGBTQI2S community in focus. 2020. https://egale.ca/wp-content/uploa ds/2020/04/Impact-of-COVID-19-Canada\%E2\%80\%99s-LGBTQ I2S-Community-in-Focus-2020-04-06.pdf. Accessed 6 April 2020.

24. Tan DH, Hull MW, Yoong D, Tremblay C, O'Byrne P, Thomas $\mathrm{R}$, et al. Canadian guideline on HIV pre-exposure prophylaxis and nonoccupational postexposure prophylaxis. CMAJ. 2017;189(47):E1448-E14581458.

25. Wilton, J. \& Broechaert, L. The HIV treatment cascade: patching the leaks to improve HIV prevention. The Body Pro. 2013. https ://www.thebodypro.com/article/the-hiv-treatment-cascade-patch ing-the-leaks-to-i.

Publisher's Note Springer Nature remains neutral with regard to jurisdictional claims in published maps and institutional affiliations. 